\title{
The Use of Precautionary Loss Functions in Risk Analysis
}

\author{
Jan Gerhard Norstrom \\ Delft University of Technology, Delft
}

Key Words - Risk analysis, Loss function, Bayes estimation, Decision theory.

Summary \& Conclusions - Risk analysis is discussed within a Bayes framework. Traditionally, Bayes parameter estimation is based on a quadratic loss-function. This paper introduces an alternative asymmetric precautionary loss-function, derives its main features, and presents a general class of precautionary lossfunctions with the quadratic loss-function as a special case. These loss functions approach infinity near the origin to prevent underestimates and thus give conservative estimates, especially when, for example, low failure rates are being estimated. The conservative estimates make these loss functions useful when the consequences are major and under-estimation is serious. They are intuitively appealing and easy to calculate.

\section{INTRODUCTION}

Risk analysis is used in several industrial disciplines, $e g$, chemical-process industry and offshore industry. In risk analysis, both the potentiality of an undesired event and its consequences are investigated. This potentiality is usually measured by either a probability or a failure rate. The Bayes approach is widely applied to estimate this probability (failure rate). Some examples on the Bayes approach are in [1].

When dealing with disastrous consequences, it can be worse to underestimate the potentiality of an event than to overestimate it. This is important when risk-level is the basis of a risk-reducing initiative, either by reducing the potentiality or the consequences. An erroneously low estimated risk-level can lead to the absence of necessary initiative to reduce the risk level. It is unreasonable to use a loss function that allows one to estimate a failure probability of zero. A positive loss function at the origin allows estimating zero, and in a risk analysis, estimating zero failure probability simply means that no risk is anticipated. Hence, a precautionary loss function is defined, a specific class of loss functions that are precautionary is developed. Some examples are given. The uses of Bayes models are described in $[2,3,5,6]$.

\section{Notation}

$\Omega \quad$ sample space

$\boldsymbol{x} \quad\left(x_{1}, \ldots x_{n}\right): n \times 1$ vector of $s$-independent observations of the r.v.; $x \in \Omega$

$\theta \quad$ space of all possible $\theta$ describing the potentiality of the event

$A$ space of estimates $a \in A$ of the unknown $\theta \in \Theta$

$l(\theta, a)$ loss function: cost of estimating $a$ when $\theta$ is the value $\pi(\theta) \quad$ prior $\operatorname{pdf}\{\theta\}$

$f\left(x_{i} \mid \theta\right) \quad \operatorname{pdf}\left\{X_{i} \mid \theta\right\}$

$f(x \mid \theta) \quad \prod_{i} f\left(x_{i} \mid \theta\right):$ joint $\operatorname{pdf}\{X\}$

$\pi(\theta \mid x)$ posterior $\operatorname{pdf}\{\theta \mid x\}$

$k \quad$ precautionary index.

Other, standard notation is given in "Information for Readers \& Authors" at the rear of each issue.

\section{LOSS FUNCTION}

Optimal policy selection has traditionally been discussed in relation to symmetric (and often quadratic) loss functions. By using non-symmetric loss functions one is able to deal with cases where it is more damaging to miss the target on one side than the other. According to [3], should $l(\theta, a)$ be continuous, quasi-convex ${ }^{l}$ and attain the lowest value at $a=\theta$. This implies that the loss function increases as $a$ moves away from $\theta$.

A loss function is (for any $\epsilon>0$ ):

- downside damaging if $l(a-\epsilon, a) \geq l(a+\epsilon, a)$,

- upside damaging if $l(a-\epsilon, a) \leq l(a+\epsilon, a)$,

- symmetric if the loss function is both downside and upside damaging.

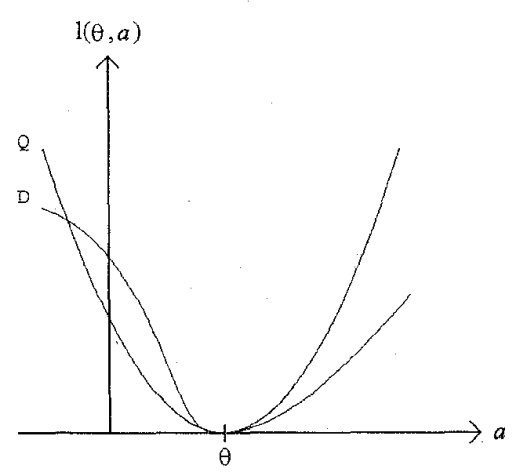

Figure 1. Functional Form of an Asymmetric DownsideDamaging Loss Function ( $D$ ) vs a Quadratic Loss Function (Q)

Figure 1 is an example of a downside-damaging loss function compared with a quadratic loss function. The downside-

\footnotetext{
${ }^{1}$ A real function $h(x)$ is quasi-convex if, for any given real number $r$, the set of all $x$ such that $h(x) \leq r$ is convex. Any convex function is quasi-convex, but the converse is not necessarily true.
} 
damaging loss function gives higher costs for an underestimate compared to the quadratic loss function.

\subsection{Precautionary Loss Functions}

The $l(\theta, a)$ is a precautionary loss function iff:

1. $l(\theta, a)$ is downside damaging, and

2. for each fixed $\theta, l(\theta, a) \rightarrow \infty$ when $a \rightarrow 0$.

Figure 2 shows some typical precautionary loss functions for $k>0$.

\subsection{Class of Precautionary Loss Functions}

A literature search for precautionary loss functions gave some variants of,

$l(\theta, a)=(\ln (a / \theta))^{2}$,

which is mentioned in [6]. The Bayes' estimate for (1) is usually difficult to calculate. The problem is to come up with a loss function which gives both simple calculations and is intuitively appealing, eg,

$l(\theta, a)=(\theta-a)^{2} / a$.

This satisfies the criterion of being precautionary. The Bayes' estimate for (2) is obtained from theorem 1.

Theorem 1. Let $l(\theta, a)$ be defined by (2). The Bayes' estimate is:

$\hat{\theta}_{p}^{2}=E_{\pi(\theta \mid x)}\left\{\theta^{2}\right\}=\int_{\theta \in \theta} \theta^{2} \cdot \pi(\theta \mid x) d \theta$

$\hat{\theta_{p}}=f \cdot E_{\pi(\theta \mid x)}\{\theta\}$,

$$
f^{2} \equiv 1+\operatorname{Var}_{\pi(\theta \mid x)}\{\theta\} / E_{\pi(\theta \mid x)}^{2}\{\theta\}>1 .
$$

Theorem 1 is proved in [4]. There it is also shown that the $s$-expected squared estimation error is less than twice the posterior variance. The Bayes' estimate in (3) is a geometric mean; in (4) it is given as a function of the posterior $s$-expectation and the posterior variance. The $f$ represents the state of being precautionary, and $f$ becomes important if the $s$-expectation is close to zero or when the variance is large. It means that the Bayes' estimate is sensitive to the choice of the loss function when very uncertain conditions occur or when estimating low probabilities.

The loss function in (2) can be generalized to

$l(\theta, a)=\frac{(\theta-a)^{2}}{a^{k}} \cdot w(\theta), 0<k \leq 2, w(\theta)>0 ;$

$w(\theta)$ is an arbitrary weight function. The $k \leq 2$ ensures that the cost increases as the difference $a-\theta$ grows. $k$ is a precautionary index since it regulates how downside damaging the loss function is. The loss function in (5) covers a spectrum of precautionary loss functions. Figure 2 plots (5) for various $k$.

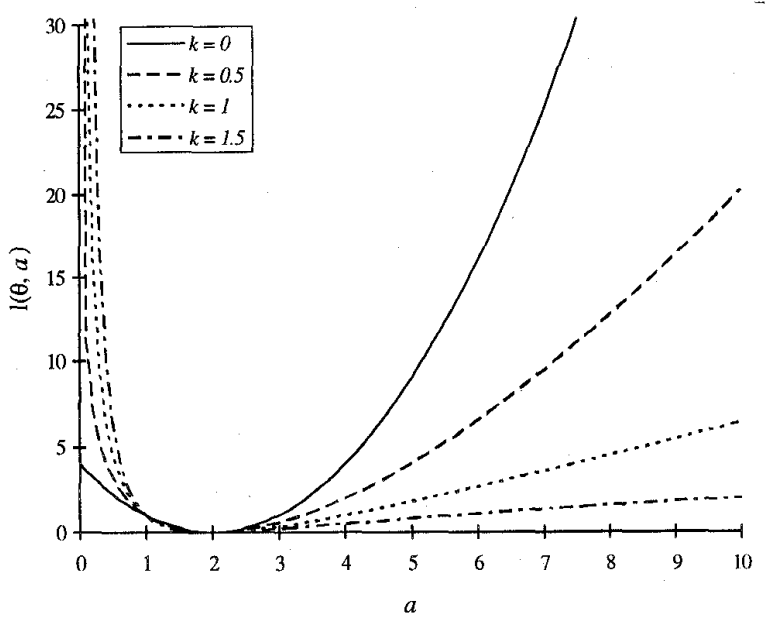

Figure 2. The Loss Function (5) vs $k$

In the limit, $k=0$, the loss function is the familiar quadratic loss function, and when $k=2$ it approaches $w(\theta)$ as $a \rightarrow \infty$. Thus, the loss function becomes more precautionary as $k$ increases.

A way to determine $k$ is to:

- select two values of $a, \theta-\epsilon$, and $\theta+c \cdot \epsilon$, where $c>1$, which have equal loss for the decision maker,

- solve $l(\theta, \theta-\epsilon)=l(\theta, \theta+c \cdot \epsilon)$ with respect to $k$.

Let $\epsilon=\theta / c$; the problem simplifies to:

$l(\theta, \theta-\theta / c)=l(\theta, 2 \theta)$

solving this with respect to $k$ gives:

$k=2 \cdot \ln (c) / \ln [2 c /(c-1)]$.

Two special values of $c$ are:

- $c=2$ gives $k=1$ as in (2),

- $c=3$ gives $k=2$.

Theorem 2. Let the loss function be defined by (5). Then the Bayes' estimate is:

$$
\begin{aligned}
& \hat{\theta}_{p}=\frac{1}{\Psi_{1}(x)} \cdot\left[\bar{k}+\sqrt{\bar{k}^{2}+\Psi_{1}(x) \cdot \Psi_{2}(x)}\right], \\
& \Psi_{1}(x) \equiv(1+\bar{k}) \cdot E_{\pi(\theta \mid x)}\{w(\theta)\} / E_{\pi(\theta \mid x)}\{\theta \cdot w(\theta)\}, \\
& \Psi_{2}(x) \equiv(1-\bar{k}) \cdot E_{\pi(\theta \mid x)}\left\{\theta^{2} \cdot w(\theta)\right\} / E_{\pi(\theta \mid x)}\{\theta \cdot w(\theta)\}, \\
& \bar{k} \equiv 1-k .
\end{aligned}
$$

The proof is obtained by differentiating the posterior $s$-expected loss and equating it to zero; then solving this for $a$ to find the critical points. Since $a>0$, the sign in front of the square root is positive. Since $a$ is the action with minimum loss and therefore is the Bayes' estimate, it is denoted $\hat{\theta}_{p}$ and the theorem is proved. 

$=1$.

Theorem 1 follows from theorem 2 for $k=1$ and $w(\theta)$

\section{EXAMPLES ${ }^{2}$}

Two examples apply the precautionary loss function. Example 1 gives a theoretical illustration while example 2 is a numerical case. The estimates are compared with those obtained from the quadratic loss function. We consider the Poisson sample model and use only the precautionary loss function with $k=1, w(\theta)=1$, and a conjugate prior.

\section{Notation}

$\lambda \quad$ unknown failure rate, to be estimated.

\subsection{Example 1: Poisson}

The Poisson sampling model of $s$ failures in $t$ time is:

$f(s \mid \lambda ; t)=\operatorname{poim}(s ; \lambda \cdot t)$

The conjugate prior in this case is the gamma pdf:

$\pi(\lambda ; \alpha, \beta)=\beta \cdot \operatorname{gamd}(\beta \cdot \lambda ; \alpha)$

Bayes' theorem gives the posterior gamma pdf:

$\pi(\lambda \mid s ; t, \alpha, \beta)=(t+\beta) \cdot \operatorname{gamd}((t+\beta) \cdot \lambda ; s+\alpha)$,

given that $s$ failures in time $t$ are observed (here $\lambda \equiv \theta$ ). The posterior $s$-expectation \& variance are:

$\hat{\lambda_{q}}=(s+\alpha) /(t+\beta)$,

$\operatorname{Var}=(s+\alpha) /(t+\beta)^{2}$.

The quadratic loss function gives the $\hat{\lambda}_{q}$ as the Bayes' estimate. This is compared with the Bayes' estimate obtained by using the precautionary loss function with $k=1$ :

$$
\begin{aligned}
& \hat{\lambda_{p}}=\frac{\sqrt{(s+\alpha) \cdot(s+\alpha+1)}}{t+\beta} \\
&=\hat{\lambda_{q}} \cdot f \\
& f \equiv \sqrt{1+\frac{1}{s+\alpha}} ; \\
& f \rightarrow 1 \text { as } s \text { increases }- \text { as shown in figure } 3 .
\end{aligned}
$$

\footnotetext{
${ }^{2}$ The number of significant figures is not intended to imply any accuracy in the estimates, but to illustrate the arithmetic.
}

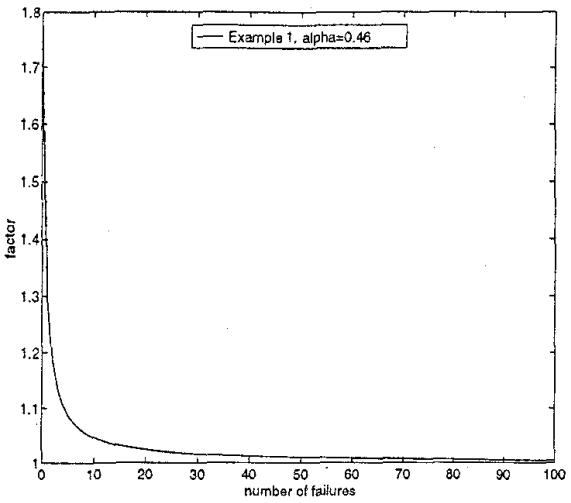

Figure 3. How $f \rightarrow 1$ as $s$ increases

\subsection{Example 2}

Some offshore reliability data are used to illustrate the effect of the precautionary loss function. When a prior data-set is available, empirical Bayes is used to model the failure frequency of a pump. This has resulted in a posterior gamma distribution with:

$s+\alpha=1.31$,

$t+\beta=25300$

in (9). These values give the posterior mean and variance:

$\hat{\lambda}_{q}=51.7 \cdot 10^{-6} /$ hour

$\operatorname{Var}=20.5 \cdot 10^{-10} /$ hour $^{2}$.

The Bayes estimate for the quadratic loss function is:

$\hat{\lambda_{q}}=51.7 \cdot 10^{-6} /$ hour

and the Bayes estimate from theorem 1 is, from (10):

$$
\begin{aligned}
\hat{\lambda_{p}} & =f \cdot \hat{\lambda_{q}}=1.33 \cdot 51.7 \cdot 10^{-6} / \text { hour } \\
& =68.7 \cdot 10^{-6} / \text { hour }=0.60 / \text { year } .
\end{aligned}
$$

This means that the Bayes estimate increases by $33 \%$ when the precautionary loss function is used instead of the quadratic loss function.

\section{ACKNOWLEDGMENT}

This work was carried out while I was at The Norwegian University of Science änd Technology (NTNU). I am pleased to thank Per Hokstad of SINTEF Safety and Reliability who encouraged me and suggested useful improvements. The research for this paper was supported by the Growth Point Centre in Safety and Reliability at SINTEF and the NTNU. 


\section{REFERENCES}

[1] G. Apostolakis, "Data specialization for plant specific risk studies", Nuclear Engineering and Design, vol 56, 1980, pp 321-329.

[2] J.O. Berger, Statistical Decision Theory and Bayesian Analysis, 1988; Springer.

[3] Y. Hosomatsu, "Concepts, theory, and techniques. Asymmetric loss function and optimal policy under uncertainty: A simple proof', Management Science, vol 26, 1980 May, pp 577-585.

[4] J.G. Norstrøm, "Bayesian analysis \& decision theory in reliability and risk analysis", Diploma Thesis, 1994 Feb, pp 33-43; The Norwegian University of Science and Technology.

[5] H.F. Martz, R.A. Waller, Bayesian Reliability Analysis, 1982; John Wiley $\&$ Sons.

[6] F.A. Tillman, W. Kuo, C.L. Hwang, L.G. Doris, "Bayesian reliability \& availability \% A review”, IEEE Trans. Reliability, vol R-31, 1982 Oct, pp 362-372.

\section{AUTHOR}

Jan G. Norstrøm; Dept. of Statistics, Probability, and Operations Research; Delft University of Technology; Mekelweg 4; 2628 CD Delft, The NETHERLANDS.

Internet (e-mail): j.g.norstrom@twi.tudelft.nl

Jan G. Norstrom holds an MSc from The Norwegian University of Science and Technology (NTNU) in Trondheim. After graduating from NTNU in 1994 he joined the young graduate training program of the European Space Agency (ESA) and spent one year in the Reliability, Availability, Maintainability \& Safety Section at the Research and Technical Centre of ESA where he worked with development of sneak-path analysis for computer-controlled systems. Since 1996 January he has been a PhD candidate at the Delft University of Technology. His research interests are applications of decision analysis within the area of risk \& reliability analysis.

Manuscript received 1996 May 20

Publisher Item Identifier S 0018-9529(96)07358-7

\section{Invitation to Membership in the Reliability Society}

There is no better time than now to join the IEEE Reliability Society. Membership gives you ready access to meetings and conferences in your area of interest, and to the prime movers in engineering, science, and business.

As an IEEE member, your can choose from a wide offering of standards, products, and services (books, conference records, employment surveys, short courses, and other helpful aids) - all at reduced member rates. Your membership entitles you to reduced registration fees for most activities sponsored or cosponsored by the IEEE and/or Reliability Society. This could easily save you more than the cost of annual membership.
Active local Reliability Society Chapters are in many locations in the USA and in Canada (Montreal, Ottawa), Japan (Tokyo), and Rep. of Singapore. The chapters offer opportunities for your personal participation and growth. Association with other Reliability Society members helps you to exchange information \& experience on current technical \& management problems and to learn how others are handling them.

Don't wait. If you are already an IEEE member, just send in your Reliability Society fee to the IEEE. If you are not an IEEE member, then write or call IEEE for an IEEE membership application.

There are 2 areas in the IEEE Reliability Society; you can join either one or both. The fee for either is $\$ 20 /$ year; the fee for both is $\$ 30 /$ year. Everyone gets the Society Newsletter. The other publication benefits are:

Area
IEEE Identification
IEEE Transactions on
Annual Proceedings

\section{Area}

Annual Proceedings

\author{
System Reliability \\ $007 / 1091$ \\ Reliability
}

Ann. Reliability \& Maintainability Symp.
Parts, Physics of Failure $007 / 1451$

Semiconductor Manufacturing Int'l Reliability Physics Symp.

IEEE Service Ctr; POBox 1331; Piscataway, NJ 08855 USA. phone: [1] 908-981-1393, or 800-678-IEEE (USA only) If you are a Reliability Society member, show this invitation to a colleague — sign up another member. $\rightarrow$ TR 